\title{
Predictors of false-positive stroke thrombectomy transfers
}

\author{
Julia Yi, ${ }^{1}$ Danielle Zielinski, ${ }^{2}$ Bichun Ouyang, ${ }^{3}$ James Conners, ${ }^{3}$ Rima Dafer, ${ }^{3}$ \\ Michael Chen ${ }^{2}$
}

${ }^{1}$ University of Illinois at Chicago, Chicago, Illinois, USA ${ }^{2}$ Department of Neurological Surgery, Rush University Medical Center, Chicago, Illinois, USA ${ }^{3}$ Department of Neurological Sciences, Rush University Medical Center, Chicago, Illinois, USA

Correspondence to Dr Michael Chen, 1725 West Harrison Street, Suite 855 Chicago, IL 60612, USA; michael_chen@rush.edu

Received 18 February 2017 Revised 7 March 2017 Accepted 8 March 2017 Published Online First 30 March 2017

\section{ABSTRACT}

Background Most patients with large vessel occlusion (LVO) stroke need to be transferred to receive thrombectomy. To save time, the decision to transfer often relies on clinical scales as a surrogate for LVO rather than imaging. However, clinical scales have been associated with high levels of diagnostic error. The aim of this study is to define the susceptibility to overdiagnosis of our current transfer decision process by measuring the rate of non-treatment transfers, the most common reasons for no treatment and potential predictors.

Methods Clinical and transfer data on consecutive patients transferred to a single endovascular capable centre for possible thrombectomy via stroke code activation were retrospectively reviewed. Whether patients underwent the procedure, why they did not undergo the procedure, and other clinical and logistical predictors were recorded. $\chi^{2}$ tests and multivariate logistic regression analysis were performed.

Results From 2015 to 2016, 105/192 transferred patients (54\%) did not undergo thrombectomy and the most common reason was absence of a LVO found on CTA after transfer (71/104 (68\%)). 14/16 (88\%) with a National Institutes of Health Stroke Scale (NIHSS) score $<10$ did not undergo thrombectomy while 41/78 (52\%) with a NIHSS $>20$ underwent thrombectomy $(p<0.001)$. Helicopter use was associated with no treatment $(p=0.004)$ while arrival within 5 hours was associated with treatment $(p<0.001)$.

Conclusions Clinical scales appear to overdiagnose LVO and may be responsible for the majority of our stroke code transfers not undergoing thrombectomy. Primary stroke centres therefore have reason to develop the capability to rapidly acquire and interpret a CTA in patients with suspected LVO prior to transfer. Such efforts may reduce the costs associated with unnecessary thrombectomy transfers.

\section{INTRODUCTION}

For nearly 20 years, hospitals caring for patients with acute stroke have been organised to deliver intravenous thrombolysis. While robust level 1a evidence was published in 2015 proving clinical benefit for mechanical thrombectomy in acute anterior circulation large vessel occlusions (LVOs), ${ }^{1-3}$ only about $10 \%$ of the 1111 primary stroke centres in the US are able to reliably provide mechanical thrombectomy. As a result, most patients initially evaluated and deemed to have a LVO need to be transferred.
Over the last 2 years, our stroke team has noticed a large number of thrombectomy transfers who do not end up undergoing the procedure. To minimise time to recanalisation, the decision to transfer a patient for thrombectomy is often based on the severity of the clinical exam rather than brain vascular imaging. As suggested by American Heart Association (AHA) guidelines, National Institutes of Health Stroke Scale score (NIHSS) $>6$ is often used as a surrogate marker for the presence of a LVO. ${ }^{4}$ Turc et al ${ }^{5}$ evaluated the ability of 13 clinical scores to predict large artery occlusions in over 1000 patients and found higher than expected false negative and false positive rates. Furthermore, patient transfers for potential thrombectomy have considerable costs, which include, but are not limited to, ambulance/helicopter services, call-pay for the neuroendovascular and stroke neurology team, repeat imaging, and a temporary suspension of what all members of the team are doing to await patient arrival. ${ }^{6}$ Sonig et $a l^{7}$ evaluated 1311511 National Inpatient Sample stroke admissions from 2008 to 2010 and found that each transferred patient undergoing tissue plasminogen activator (tPA) and thrombectomy had a mean expenditure of $\$ 27000$ more than those who were not transferred. Thus, despite the potential to incur significant unnecessary costs, the decision to transfer is currently based on an oblique measure of a LVO.

In this study, we measure the frequency of nontreatment transfers since the publication of the positive thrombectomy trials and ascertain the most common reasons why transferred patients do not undergo thrombectomy. Our study objective is to find potential predictors of non-treatment transfers based on the limited clinical information available during the initial assessment at the transferring hospital. In doing so, we may be able to tailor strategies to improve the transfer decision process.

\section{METHODS}

\section{Stroke transfer process}

The stroke service is staffed with five stroke neurologists who provide 24/7/365 coverage via a dedicated telephone hotline or telestroke. The initial determination for each consultation is eligibility for intravenous thrombolysis. If there is clinical suspicion for a disabling stroke due to a LVO, arrangements are made for transfer. Considerations for transfer include NIHSS $>6$; neurological examination findings suggestive of cortical involvement, such as gaze preference, neglect, or motor deficit with language impairment; onset of symptoms

\footnotetext{
To cite: Yi J, Zielinski

Ouyang $\mathrm{B}$, et al.

J Neurolntervent Surg

2017;9:834-836.
} 
$<6$ hours; and initial head CT with an Alberta Stroke Program Early CT score (ASPECTS) score of $\geq 6$, or a dense middle cerebral artery or basilar sign suspicious of a LVO. Patients with serious medical comorbidities including severe dementia, terminal illness or pre-stroke modified Rankin scale $\geq 3$ are usually not transferred. Conventionally, brain vascular imaging has not been recommended at the transferring hospital because of how unpredictable and potentially time consuming it can be for acquisition and interpretation. If the stroke neurologist deems the patient a candidate for thrombectomy, after discussion with the neurointerventionalist, the stroke code group page is activated and relevant team members aim to meet at the emergency department bay prior to the patient's arrival. Every stroke code group page activation constituted the denominator for this study. Upon arrival, the patient's neurological exam is reassessed by the stroke team. If a disabling deficit persists, brain parenchymal and vascular imaging are immediately obtained to determine if the ASPECTS score is $\geq 6$ and confirm a LVO prior to the decision to proceed with thrombectomy.

\section{Data collection}

Institutional review board approval was not necessary because the data were obtained as part of a collaborative quality improvement project for our hospital stroke certification programme. Records of consecutive patients transferred to our single, urban, tertiary care academic medical centre were reviewed. Every stroke transfer is coordinated through the hospital transfer centre, which records demographical data on the patient. Reports of all transfers are then sent to the stroke programme data analyst who then adds additional predetermined clinical information from our electronic hospital medical system (Epic) for the purposes of hospital stroke programme credentialing. An on-call advanced practice nurse practitioner also responds to every stroke alert and records details of the transfer - time stamps for hospital and imaging, and the reason why the patient did not undergo thrombectomy, if applicable. These data were prospectively recorded and serve as the source of data for this report. Date, patient age, method of transportation, initial NIHSS at transferring hospital, telestroke video use, whether the transfer call was placed after hours, whether the patient arrived at our hospital within 5 hours since last known well and whether the referring hospital was a primary stroke centre were recorded as independent variables. Outcomes included the total number of transfers, the number of non-treatment transfers, and the reasons why these patients were not treated. $\chi^{2}$ tests and multivariate logistic regression analysis compared demographical and clinical variables, including age and NIHSS among others, between patients with and without thrombectomy. All statistical analyses were performed with SAS 9.3 (SAS Institute Inc, Cary, North Carolina, USA). A p value $<0.05$ was considered significant.

\section{RESULTS}

Between January 2015 and December 2016, 105 out of the 192 potential thrombectomy transfers (54\%) did not undergo thrombectomy. Brain vascular imaging-usually CTA-upon arrival showing no LVO was by far the most common reason for not undergoing thrombectomy (71/104 (68\%)). An improving neurological examination and an ASPECTS score $<6$ were the two additional most common but far less common reasons for no treatment (table 1 ).

Nearly a third of all transfers used air transport and occurred during the evening hours. Half of transfers were evaluated first with telestroke video. The majority of patients received
Table 1 Reasons for no treatment among consecutive transfers for mechanical thrombectomy

\begin{tabular}{lc} 
Variable & Total $\mathbf{n = 1 0 4}$ \\
\hline No large vessel occlusion & $71(67 \%)$ \\
Significant exam improvement & $19(18 \%)$ \\
ASPECTS $<6$ & $10(10 \%)$ \\
Premorbid disability discovered & $3(3 \%)$ \\
Patient unstable & $2(2 \%)$ \\
\hline
\end{tabular}

ASPECTS, Alberta Stroke Program Early CT score.

Table 2 Predictors of no treatment among consecutive transfers for mechanical thrombectomy

\begin{tabular}{lcccl}
\hline Variable & $\begin{array}{l}\text { Total } \\
\mathrm{n}=192\end{array}$ & $\begin{array}{l}\text { No IA } \\
\mathrm{n}=104\end{array}$ & IA n=88 & p Value* \\
\hline Age, mean (SD) & $67.3(14.0)$ & $66.8(13.9)$ & $67.8(14.2)$ & 0.85 \\
Age>80 & $42(21.9 \%)$ & $18(17.3 \%)$ & $24(27.3 \%)$ & 0.1 \\
Helicopter & $67(34.9 \%)$ & $46(44.2 \%)$ & $21(23.9 \%)$ & 0.003 \\
Intravenous tPA given & $140(72.9 \%)$ & $73(70.2 \%)$ & $67(76.1 \%)$ & 0.36 \\
NIHSS & & & & 0.01 \\
$\quad<10$ & $16(8.3 \%)$ & $14(13.5 \%)$ & $2(2.3 \%)$ & \\
$\quad 10-20$ & $98(51.0 \%)$ & $53(51.0 \%)$ & $45(51.1 \%)$ & \\
$>20$ & $78(40.6 \%)$ & $37(35.6 \%)$ & $41(46.6 \%)$ & \\
Mean (SD) & $18.8(6.2)$ & $17.5(6.8)$ & $20.5(4.8)$ & 0.0005 \\
Telestroke video used & $94(49.0 \%)$ & $53(51.0 \%)$ & $41(46.6 \%)$ & 0.55 \\
19:00 to 07:00 & $69(35.9 \%)$ & $33(31.7 \%)$ & $36(40.9 \%)$ & 0.19 \\
Arrival within 5 hours & $136(70.8 \%)$ & $63(60.6 \%)$ & $73(83.0 \%)$ & 0.001 \\
From primary stroke & $154(80.2 \%)$ & $81(77.9 \%)$ & $73(83.0 \%)$ & 0.38 \\
centre & & & & \\
\hline IA, intra-arterial; NIHSS, National Institutes of Health Stroke Scale; tPA, tissue \\
plasminogen activator.
\end{tabular}

intravenous thrombolysis, arrived within 5 hours and came from a Joint Commission certified primary stroke centre.

The strongest predictor of no treatment was the initial NIHSS; $14 / 16(88 \%)$ with a NIHSS $<10$ did not undergo thrombectomy, while $41 / 78(52 \%)$ with a NIHSS $>20$ underwent thrombectomy $(\mathrm{p}<0.001)$. Helicopter use was significantly associated with no treatment $(\mathrm{p}=0.004)$. Arrival within 5 hours was significantly associated with treatment $(\mathrm{p}<0.001)$ (table 2$)$. When used as a continuous variable, NIHSS, along with helicopter use and arrival within 5 hours, were all predictors in multivariate logistic regression analysis (table 3 ).

\section{DISCUSSION}

Our results show that more than half of our potential thrombectomy transfers since January 2015 did not end up undergoing thrombectomy. The most common reason (67\%) for a nontreatment transfer was brain vascular imaging after transfer showing absence of a LVO. A minority of patients did not undergo thrombectomy because of a significant exam improvement or ASPECTS $<6$. Because the majority of patients received intravenous thrombolysis prior to transfer (72\%), an improving exam could be explained by vessel recanalisation while en route. However, intravenous tPA effectively recanalises LVOs only a minority of times, with larger and longer clots shown to be recalcitrant. ${ }^{8} 9$ Hence, there may be reason to believe that an 
Table 3 Multivariate logistic regression

\begin{tabular}{lll}
\hline Variable & OR $(95 \%$ Cl $)$ & $\mathbf{p}$ Value \\
\hline Age $>=80$ & $1.82(0.84$ to 3.92$)$ & 0.13 \\
NIHSS & $1.09(1.03$ to 1.15$)$ & 0.004 \\
Intravenous tPA & $0.79(0.35$ to 1.77$)$ & 0.57 \\
Helicopter & $0.29(0.14$ to 0.61$)$ & 0.001 \\
Telestroke video used & $1.01(0.53$ to 1.92$)$ & 0.97 \\
19:00 to 07:00 & $1.33(0.69$ to 2.59$)$ & 0.40 \\
Arrival within 5 hours & $4.28(1.88$ to 9.71$)$ & 0.0005 \\
From primary stroke centre & $1.29(0.56$ to 2.95$)$ & 0.55 \\
\hline
\end{tabular}

even larger proportion of the patients transferred for suspected LVO based on clinical exam and scales may not have had one in the first place.

Prior studies reported up to a third of transfers demonstrated 'ASPECTS decay' or rapid progression of cerebral infarction during transfer. A high NIHSS on admission was found to be a predictor. ${ }^{1011}$ A non-contrast head CT is a standard part of our evaluation after transfer and we found a lower than expected $(10 \%)$ rate of ASPECTS $<6$ as a reason not to undergo thrombectomy. This discrepancy may be explained by an increasingly faster transfer process than our more recent data reflect compared with prior publications. As the many components of stroke transfers continue to become more integrated with time, infarct progression during lengthy transportation will be less common.

The time of day, telestroke use, and whether the transfer originated from a primary stroke centre did not appear to affect the rate of overdiagnosis. We identified three predictors of whether the patient underwent treatment: helicopter use, patient arrival within 5 hours and NIHSS severity on admission. Helicopter transportation costs alone can range from $\$ 12000$ to $\$ 25000$ and two-thirds of patients who arrived in this fashion did not undergo thrombectomy. One explanation for this finding that may be unique to our hospital is its urban location and the location of the helipad being two city blocks away. A separate ambulance transfer service is necessary to transport the patient after landing to our hospital. Nevertheless, this association of no treatment with helicopter use warrants additional investigation, including the possibility of ASPECTS decay as a mechanism. NIHSS $<10$ at the transferring hospital reliably predicted a non-treatment transfer. Therefore, it may be particularly worthwhile in this subgroup to take the added effort and time to obtain a CTA prior to transfer. Despite the AHA recommendation to use NIHSS $>6^{4}$ to select patients for LVO, our observed trends over the past 2 years are more consistent with the results by Turc et al,,$^{5}$ showing that modification of the NIHSS cutoff below a threshold of NIHSS $>11$ would result in sending almost every stroke patient to an endovascular capable centre. Although NIHSS $<10$ was predictive, NIHSS 10-20 $(50 \%)$ and NIHSS $>20(36 \%)$ had a significant proportion of patients not undergoing thrombectomy.

The ability to rapidly obtain and interpret brain vascular imaging not only catalyses the treatment process for patients with actual LVOs, but also, as our data suggest, could reduce the number of non-treatment transfers by reliably detecting the most common reason for not undergoing a procedure. One concern for obtaining a CTA at the transferring hospital is the unpredictability of acquisition and interpretation. Liang et $a l^{12}$ demonstrated the feasibility of outreach efforts to improve the performance of transferring hospitals in rapidly obtaining a CTA prior to transfer. After their efforts, among 57 consecutive transfers, stroke onset to groin puncture time was similar regardless of whether CTA was done at the transferring or hospital or the endovascular-capable hospital. Another concern for the use of CTA to select patients prior to transfer.

Some challenges consist of having the local expertise available to interpret the CTA immediately after acquisition. Telestroke may, particularly for images that can be automatically and immediately uploaded, allow for rapid CTA interpretation by the receiving hospital prior to the decision to transfer. Anecdotally, some stroke neurologists ask the physician at the transferring hospital to take mobile phone videos or snapshots of the CTA as the images are being acquired to save time. The ability of stroke neurologists to reliably and accurately interpret a CTA brain to detect LVO is therefore obviously critical in this time-sensitive process that demands instant availability. Bar et $a l^{13}$ validated this practice with strong inter-rater agreement in the CTA evaluation for occlusion in 75 patients with acute stroke between stroke neurologists and neuroradiologists. This study lends data to practices that are likely already in place to improve triage efficiencies.

Limitations to our study consist of the variability in clinical examination methods to determine stroke severity. Among the five stroke neurologists involved with the transfer decision process over the past 2 years, there was no uniform agreement on which clinical scale to use or what cutoff to use as a screen for a LVO. Perhaps use of standardised criteria would have yielded a lower number of non-treatment transfers. However, half of transfers did not involve telestroke, and it may be challenging to achieve an accurate, consistent neurological assessment with an emergency department physician, often in the middle of the night, over the telephone.

Another concern is that transfer of patients with LVOs to a comprehensive stroke centre may have benefits for the patient in addition to thrombectomy. Advanced neurocritical and neurosurgical care may not be available at the transferring hospital, and potentially justify the transfer. However, our data suggest that since $67 \%$ of non-treatment transfers lack LVO, these patients may in fact be effectively managed without advanced neurocritical and/or neurosurgical care.

Endovascular capable centres should reconsider accepting a significant rate of overdiagnosis in patients with stroke who are considered potentially thrombolysable prior to transfer. Overdiagnosis in this fashion is costly, and as our data suggest, may be effectively minimised with a brain CTA prior to transfer. These findings justify efforts to develop and potentially mandate workflow at the over 1000 primary stroke centres to be able to quickly acquire and interpret a CTA brain to efficiently and accurately diagnose a LVO as early as possible. If such efforts can be realised, much like other workflow goals mandated for delivery of intravenous tPA, improved diagnostic accuracy could be obtained while minimising elapsed time and the costs associated with unnecessary transfers. Our data suggest that the use of clinical scales, in particular patients with NIHSS $<10$, may result in unnecessary thrombectomy transfers which could be obviated with a CTA prior to transfer.

\section{Acknowledgements Renata Costa.}

Contributors All listed authors ( JY, DZ, BO, JC, RD and MC) contributed to the design, data collection, data analysis and drafting of the manuscript.

Competing interests $\mathrm{MC}$ is a consultant for Genentech, Penumbra, Stryker and Medtronic.

Provenance and peer review Not commissioned; externally peer reviewed.

Data sharing statement The authors agree to share any data on request. 


\section{REFERENCES}

1 Berkhemer OA, Fransen PS, Beumer D, et al. A randomized trial of intraarterial treatment for acute ischemic stroke. N Engl J Med 2015;372:11-20.

2 Campbell BC, Mitchell PJ, Kleinig TJ, et al. Endovascular therapy for ischemic stroke with perfusion-imaging selection. N Engl J Med 2015;372:1009-18.

3 Goyal M, Demchuk AM, Menon BK, et al. Randomized assessment of rapid endovascular treatment of ischemic stroke. N Engl J Med 2015;372:1019-30.

4 Powers WJ, Derdeyn CP, Biller J, et al. 2015 American Heart Association/American Stroke Association focused update of the 2013 guidelines for the early management of patients with acute ischemic stroke regarding endovascular treatment: a guideline for healthcare professionals from the American Heart Association/American Stroke Association. Stroke 2015:46:3020-35.

5 Turc G, Maïer B, Naggara 0, et al. Clinical scales do not reliably identify acute ischemic stroke patients with large-artery occlusion. Stroke 2016:47:1466-72.

6 Chen M. Cost-effectiveness of endovascular therapy for acute ischemic stroke. Neurology 2012;79(Suppl 1):S16-21.

7 Sonig A, Lin N, Krishna C, et al. Impact of transfer status on hospitalization cost and discharge disposition for acute ischemic stroke across the US. J Neurosurg 2016;124:1228-37.
8 Bhatia $R$, Hill MD, Shobha $N$, et al. Low rates of acute recanalization with intravenous recombinant tissue plasminogen activator in ischemic stroke: real-world experience and a call for action. Stroke 2010:41:2254-8.

9 Riedel $\mathrm{CH}$, Zimmermann $\mathrm{P}$, Jensen-Kondering $\mathrm{U}$, et al. The importance of size: successful recanalization by intravenous thrombolysis in acute anterior stroke depends on thrombus length. Stroke 2011;42:1775-7.

10 Mokin M, Gupta R, Guerrero WR, et al. ASPECTS decay during inter-facility transfer in patients with large vessel occlusion strokes. J Neurointerv Surg 2016; $\mathbf{m}$.

11 Sun CH, Connelly K, Nogueira RG, et al. ASPECTS decay during inter-facility transfer predicts patient outcomes in endovascular reperfusion for ischemic stroke: a unique assessment of dynamic physiologic change over time. J Neurointerv Surg 2015;7:22-6.

12 Liang JW, Stein L, Wilson N, et al. Timing of vessel imaging for suspected large vessel occlusions does not affect groin puncture time in transfer patients with stroke. J Neurointerv Surg 2017;

13 Bar M, Kral J, Jonszta $\mathrm{T}$, et al. Interrater variability for $\mathrm{CT}$ angiography evaluation between neurologists and neuroradiologist in acute stroke patients. $\mathrm{Br} J$ Radiol 2017:90:20160670 